INTERNATIONAL JOURNAL OF SYSTEMATIC BACTERIOLOGY

Vol. 19, No. 2 April 1969

pp. 173-181

Copyright 1969, Iowa State University Press

\title{
REGARDING NOMENC LATURAL TYPES (NOMENIFERS): \\ A PROPOSAL FOR AMENDING PRINCIPLE 11 AND RULE 9 OF THE INTERNATIONAL CODE OF NOMENCLATURE OF BACTERIA
}

\section{Mortimer P. Starr and Helen Heise}

Department of Bacteriology, University of California; Davis, California 95616, and Department of Philosophy, University of California, Santa Barbara, California 93106

ABSTRACT. Amendments are proposed to Principle 11 and Rule 9 of the International Code of Nomenclature of Bacteria which should serve to correct the present ambiguous, misleading, and erroneous language regarding nomenclatural types (nomenifers).

We have set down elsewhere (Heise and Starr 1968) our analysis of the logical status and epistemological role of nomenclatural types. Our findings are that the present language regarding nomenclatural types in Principle 11 and Rule 9 of the International Code of Nomenclature of Bacteria (1966; hereinafter, Code) is ambiguous, erroneous, and misleading. The purposes of the present proposal are (a) to request the Judicial Commission of the International Committee on Nomenclature of Bacteria to consider altering the language; (b) to offer possible amendments which we believe to be improved versions of Principle 11, Rule 9a, Rule 9c(1), and Rule $9 \mathrm{~d}(1)$; and (c) to recommend thorough review of other parts of the Code to insure conformity with these proposed amendments.

Principle 11 of the Code (p. 463) presently states:

The application of the names of taxa is determined by means of nomenclatural types (nominifers). A nomenclatural type is that constituent element of a taxon to which the name of the taxon is permanently attached, whether as the accepted name or as a synonym. Note. The phrase "constituent element of a taxon" for a species or a subspecies of bacteria is the type specimen, usually a type strain or culture. 
Explicating Principle 11, Rule 9a (Code, p. 467) presently states (in part):

For each taxon covered by this rule there shall be designated a type. The nomenclatural type (nominifer) is that constituent element of the taxon to which the name of the taxon is permanently attached. The type of a species of (sic! or) subspecies is preferably a designated type strain or in special cases it may be a description, a preserved specimen or preparation, or an illustration. The type of a genus is a species within the genus designated in conformity with Rule 9c. The type of an order or suborder, family or subfamily, tribe or subtribe, is the genus on whose name the name of the higher taxon is based. The type of a taxon higher than order is one of the contained orders. The type of a taxon should be fixed at the time of its proposal by its author.

Rule 9c(1) (Code, p. 467) presently states:

The nomenclatural type (type species) of a genus or subgenus is the name of the single species or of one of the species included when the name of the genus or subgenus was originally validly published. Note. The expression "type species" is to be used rather than "genotype" or other expressions when referring to the type species of a genus.

Rule 9d(1) (Code, pp. 468-9) presently states (in part):

The type of a species or subspecies is preferably a living strain maintained in a bacteriological laboratory, more particularly in one of the permanently established culture collections from which it would be available for study.

Note 1. For a species which cannot be maintained in laboratory cultures or for which neither type strains nor neotype strains exist, the type is the original description, preparation or illustration.

The arguments relating to the ambiguities or errors of Principle 11 and Rule 9 of the Code are laid out in greater detail elsewhere (Heise and Sta rr, 1968); here we point out more briefly what we find wrong with the parts of the Code which we suggest be amended. In some cases, we have added points not treated in the other article.

First. -- A lexicographical point: "Nomenifer" is the word 
which appears in the original article by Schopf (1960), in which that word is offered instead of "type specimen". The Code shows "nominifer", though all other writers use Schopf's original spelling. "Nomenifer" comes from the Latin for "name" (nominative singular: "nomen", , genitive singular: "nominis") and "to bear" ("ferre"). Since "nomen" is a possible root by analogy with "nomenclature", and since the term was formed with this root by Schopf and his word is not totally inconsistent with rules governing the formation of words from the Latin (according to a classicist we consulted), and since subsequent writers use "nomenifer", it seems preferable to retain the original published neologism rather than devising still another term. Hence, nomenifer.

Second. -- Schopf (1960), in introducing the term "nomenifer", seems to be using it as equivalent to "type specimen". The Code apparently extends the meaning of the term to nomenclatural types of higher categories; Schopf's language may allow such extension of the term. The explicit terminology suggested by Heise and Starr (1968) might also be used to indicate clearly a particular categoreal level of nomenifer; e.g., specific nomenifer (= type specimen = name-bearer of a species or subspecies), generic nomenifer (= type species = name-bearer of a genus or subgenus), familial nomenifer (= type genus = name-bearer of a family or subfamily), etc. However, as pointed out elsewhere (Heise and Starr, 1968), the status of nomenclatural types above the type specimen seems to be significantly different in some respects from that of type specimens themselves. All nomenifers have a common purpose, namely, to direct our attention to an entity of narrower compass than the class of which the nomenifer is the nomenifer (monotypic classes excepted). The way in which this purpose is served at categoreal levels above the species is different from the way in which this is done at the species or subspecies level. The specific nomenifer is something concrete, here meaning localized in space and time. Nomenifers above the specific nomenifer are open classes, in the logicians' sense. A family, genus, or species is an open class, that is, a class admitting of indefinitely many members of a certain kind. Such an open class cannot be had in hand; it is not localized in time and space. A nomenifer above the specific nomenifer does serve the purpose of making clear which lower taxon is definitely included in the taxon being described. 
But such lower taxa cannot be held in hand to give one first hand perceptual acquaintance, as can specific nomenifers. The point is that ideally nomenifers serve two purposes: to give an unequivocal instance of the class whose name is being defined (which nomenifers of taxa at any rank can do), and to give an unequivocal concrete instance of a taxon (which only nomenifers of the lowest rank can do). We might, therefore, conclude that the ostensive definition of the name of any higher taxon (i.e., above the species or subspecies level) must, ultimately, be the set of all the specific nomenifers which are included in that higher taxon. We must again clearly distinguish between the ostensivedefining function of the nomenifer and the name-bearing function of the nomenifer. Any higher taxon would have a number of ostensively-defining type specimens (the set of all the specific nomenifers which are included in that higher taxon), but that higher taxon would have at the next lower categoreal level only one name-bearing nomenifer.

Third. - - The application of the name of the taxon cannot be solely by means of nomenifers. By itself, the nomenifer cannot show what traits are to count as class-determining. It cannot, on the one hand, because the nomenclatural type need not be typical of the taxon being described; on the other hand, the nomenclatural type is not unequivocal in respect to (a) the significant properties being exemplified, or (b) the permissible variation in the significant properties.

These difficulties can be most perspicuously discussed in relation to the type specimen. The type specimen is, as has been said, a concrete example of the lowest taxon or, equivalently, the type specimen is an ostensive definition of the taxon's name (what one may point to in order to define the name). The problems arising with the use of ostensive definition (see Heise and Starr 1968) can be seen with a simple, everyday example. Someone, probably a child, asks what "red" means. "Red" is ostensively defined by his being shown a red object (the difficulties obtain no matter what object is chosen, so it does not matter what we choose); suppose it is an apple, and the person is told "That's red". What the person working out the meaning of the word has to do is pick out which property or properties of the object are being pointed at. He might think it was the shape, or the smoothness, or the kind of fruit, rather 
than the color. That is the first problem with any instance of ostensive definition. The second difficulty with any case of ostensive definition can also be shown with the "red" example. Suppose the learning person somehow picks out the color of the apple as the property being pointed to, then the question must arise concerning the range of colors which can be covered by this word, "red". Let's say the red of the apple is a clear blue-red; should he or should he not include pink, red-orange, orange, magenta, red-violet, purple? What about variations of saturation and brilliance? The person learning the meaning of "red" can ask for many examples of what will count as red, so that comparisons and corrections will eventually in most cases get him to the right concept, but the biologist trying to learn from the nomenifer the meaning of a species' name has only one example and, even if he had more, he would still need an intensional definition, as will now be shown.

It might seem that an indefinite number of ostensive definitions of the name of a microbial taxon could be gotten because the type specimen is (a) plural (i.e., there is more than one organism in a microbial culture, unlike say a zoological type specimen which is a single organism), and (b) live. Because there are many organisms in a microbial culture and we can in addition get an indefinite number of further organisms, we can indeed have whatever number of ostensive definitions of the name of the microbial taxon is desired; so it might be thought that an intensional definition (that is, definition in terms of properties) of the name is not needed. But this is not the case. The very fact that the microbial type specimen is plural and live requires that there be an intensional definition as well as an ostensive definition. This conclusion is supported by the following considerations.

If one subdivides the microbial culture which is the type specimen in order to proliferate cultures to serve as ostensive definitions, there may be variations among these now plural cultures. The variations may be due to the genetic heterogeneity of the original culture. Then which of the possible traits was intended as belonging to the original taxon described by the naming investigator can be determined only by reference to the description of the taxon, not from the properties of the proliferated type specimen. Even in the unlikely event that the original microbial culture were 
genetically homogeneous, the liveness of the culture and the large populations mean that there will be mutations in significant numbers. And again, one must be able to decide which traits belonged to the taxon investigated by the microbiologist who named the taxon. This decision can be made only by reference to the list of properties given as neces sary by the original investigator. In sum, ostensive defini tion even, or should we say especially, in the case of names of microbes is by itself not enough; intensional definition is also necessary. So, ideally, the investigator would state-in the original, naming publication--the organism's characteristics and an appraisal of the ones which he took to be important (including the permissible ranges of their variations), as well as putting a type specimen on file. Such description and evaluation are offered by the original inves tigator, and may be added to and amended by subsequent investigators.

Fourth. - In the second sentence of Principle 11, the use of the word "attach" to indicate the relation between the name of the taxon and the nomenclatural type may be misleading. It may be interpreted to mean that the name be longs onty to the nomenclatural type, in which case the name would be a proper name (see Heise and Starr 1968). A proper name is the name of an entity which, at that logical level, is an individual. For example, New York City is an individual city though it consists of many entities (boroughs, postal zones, neighborhoods, persons, buildings, etc.) of lower logical orders. A proper name does not entail, in a strict sense, any particular qualities--a proper name has no intension is another way of saying this. So if the name were attached only to the nomenclatural type, it would be scientifically useless because that name, being the proper name of an individual, could not be attached to any other organisms; if somehow we tried to extend the name to other organisms, we would not have any criteria on the basis of which to do so. Therefore, to show that the name applied to the nomenclatural type can be applied to other organisms of that taxon, the connection between the name and the nomenclatural type should be a different one; "associated" and "related" seem preferable to "attached".

Fifth. - - Principle 11 is in error when it says in the Note: "the phrase. . . is the type specimen". What was probably intended was that the phrase mean the type specimen. This 
misleading locution may be corrected by using some word like "means" in place of "is" (which in the Code seems to be a sign of equivalence).

Sixth. - - To avoid the possibility that the type specimen might be interpreted as having some special logical status in relation to all the other members of the taxon (which the type specimen does not have), "that" preceding "constituent element" should be replaced by "a" in Principle 11 and Rule 9a.

Seventh. - - If the type specimen is an element of the taxon, neither a name nor a description nor an illustration can be a type specimen. Neither a name nor a series of words nor a drawing (or photograph) can be an element of a biological taxon, though a description or illustration can serve in place of a type specimen. This objection applies to Rule 9a, Rule 9c (1), and Rule 9d(1).

In light of these arguments and the detailed analysis we have presented elsewhere (Heise and Starr 1968), we respectfully submit to the Judicial Commission of the Inter national Committee on Nomenclature of Bacteria the following statements as possible replacements for the language of the present Principle 11 and parts of Rule 9a, Rule 9c(1), and Rule $9 \mathrm{~d}(1)$ of the Code:

\section{Principle 11}

The application of the names of taxa is determined in part by means of nomenclatural types (nomenifers). A nomenclatural type is a constituent element of a taxon with which the name of the taxon is permanently associated, whether as the accepted name or as a synonym. That is, a taxon bears that name, or a synonym, only if the nomenifer is included in the taxon. Note. The "constituent element of a taxon" referred to above, for a species or subspecies, is the type specimen (the specific nomenifer), that is, a type strain or culture. The specific nomenifer (the nomenifer of the taxon of lowest rank) is a concrete example of that taxon or, equivalently, serves as an ostensive definition of the name of that taxon. In a hierarchical classification, the ostensive definition of the name of a higher taxon (i.e., above the species or subspecies level) is, ultimately, the set of all the specific nomenifers which are included in 
that higher taxon; but that higher taxon would have at the next lower categoreal level only one name-bearing nomenifer.

Rule 9a.

For each taxon covered by this Rule, there shall be designated a nomenclatura $L$ type. The nomenclatural type (nomenifer) is a constituent element of the taxon with which the name is permanently associated. The nomenifer serves to relate the name of that taxon to the organisms which that name designates. The nomenclatural type of a species or subspecies (the specific nomenifer) is preferably a designated type strain. In those cases where a type strain is lacking, the role of the specific nomenifer may be served by a description, a preserved specimen or preparation, or an illustration. The categoreal level of the nomenclatural type is the next lower categoreal level below the taxon of which it is the nomenclatural type. Thus, the nomenclatural type of a genus (the generic nomenifer) is a species, and the ostensive definition of the name of the genus is, ultimately, the set of all the specific nomenifers which are included in that genus; the nomenclatural type of a family (the familial nomenifer) is a genus, and the ostensive defini tion of the name of the family is, ultimately, the set of all the specific nomenifers which are included in that family; the nomenclatural type of an order (the ordinal nomenifer) is a family, and the ostensive definition of the name of the order is, ultimately, the set of all the specific nomenifers which are included in that order. The nomenclatural type of a taxon should be fixed at the time the author proposes the name of the taxon.

Rule 9c. Designation of the nomenclatural type of a genus or subgenus.

(1) The nomenclatural type of a genus or subgenus (the type species or generic nomenifer) is the single species or one of the species included when the name of the genus or subgenus was originally validly published.

Rule $9 \mathrm{~d}(1)$.

The nomenclatural type of a species or subspecies is preferably a living strain maintained in a bacteriological laboratory, more particularly in one of the permanently established culture collections from which it would be available for study. 
Note 1. For a species which cannot be maintained in laboratory culture or for which neither type strains nor neotype strains exist, the role of the type specimen is served by the original description, preparation or illustration.

\section{LITERA TURE CITED}

Heise, H. and M. P. Starr. 1968. Nomenifers: are they christened or classified? Systematic Zoology, 17:458 467.

International Code of Nomenclature of Bacteria. 1966. International Journal of Systematic Bacteriology, 16: $459-490$.

Schopf, J. M. 1960. Emphasis on holotype(?). Science, $131: 1043$.

Supported by research grant AI-08426 from the National Institute of Allergy and Infectious Diseases, United States Public Health Service, and by a Fellowship (to M. P.S.) from the John Simon Guggenheim Memorial Foundation. 